Kragujevac Journal of Mathematics

Volume 45(6) (2021), Pages 897-908.

\title{
POSITIVE SOLUTIONS FOR FIRST-ORDER NONLINEAR CAPUTO-HADAMARD FRACTIONAL RELAXATION DIFFERENTIAL EQUATIONS
}

\author{
ABDELOUAHEB ARDJOUNI ${ }^{1}$ AND AHCENE DJOUDI ${ }^{2}$
}

\begin{abstract}
This article concerns the existence and uniqueness of positive solutions of the first-order nonlinear Caputo-Hadamard fractional relaxation differential equation

$$
\left\{\begin{array}{l}
\mathfrak{D}_{1}^{\alpha}(x(t)-g(t, x(t)))+w x(t)=f(t, x(t)), 1<t \leq e, \\
x(1)=x_{0}>g\left(1, x_{0}\right)>0
\end{array}\right.
$$

where $0<\alpha \leq 1$. In the process we convert the given fractional differential equation into an equivalent integral equation. Then we construct appropriate mappings and employ the Krasnoselskii fixed point theorem and the method of upper and lower solutions to show the existence of a positive solution of this equation. We also use the Banach fixed point theorem to show the existence of a unique positive solution. Finally, an example is given to illustrate our results.
\end{abstract}

\section{INTRODUCTION}

Fractional differential equations with and without delay arise from a variety of applications including in various fields of science and engineering such as applied sciences, practical problems concerning mechanics, the engineering technique fields, economy, control systems, physics, chemistry, biology, medicine, atomic energy, information theory, harmonic oscillator, nonlinear oscillations, conservative systems, stability and instability of geodesic on Riemannian manifolds, dynamics in Hamiltonian systems, etc. In particular, problems concerning qualitative analysis of linear and nonlinear fractional differential equations with and without delay have received the attention of many authors, see [1]-[13], [16] and the references therein.

Key words and phrases. Fixed points, fractional differential equations, positive solutions, existence, uniqueness, relaxation phenomenon.

2010 Mathematics Subject Classification. Primary: 34A08. Secondary: 34A12.

DOI 10.46793/KgJMat2106.897A

Received: February 07, 2019.

Accepted: June 13, 2019. 
Zhang in [16] investigated the existence and uniqueness of positive solutions for the nonlinear fractional differential equation

$$
\left\{\begin{array}{l}
D^{\alpha} x(t)=f(t, x(t)), \quad 0<t \leq 1 \\
x(0)=0
\end{array}\right.
$$

where $D^{\alpha}$ is the standard Riemann Liouville fractional derivative of order $0<\alpha<1$ and $f:[0,1] \times[0, \infty) \rightarrow[0, \infty)$ is a given continuous function. By using the method of the upper and lower solution and cone fixed-point theorem, the author obtained the existence and uniqueness of a positive solution.

The nonlinear fractional differential equation

$$
\left\{\begin{array}{l}
{ }^{C} D^{\alpha} x(t)=f(t, x(t))+{ }^{C} D^{\alpha-1} g(t, x(t)), \quad 0<t \leq T \\
x(0)=\theta_{1}>0, \quad x^{\prime}(0)=\theta_{2}>0
\end{array}\right.
$$

has been investigated in [4], where ${ }^{C} D^{\alpha}$ is the standard Caputo's fractional derivative of order $1<\alpha \leq 2, g, f:[0, T] \times[0, \infty) \rightarrow[0, \infty)$ are given continuous functions, $g$ is non-decreasing on $x$ and $\theta_{2} \geq g\left(0, \theta_{1}\right)$. By employing the method of the upper and lower solutions and Schauder and Banach fixed point theorems, the authors obtained positivity results.

In [6], Chidouh, Guezane-Lakoud and Bebbouchi discussed the existence and uniqueness of the positive solution of the following nonlinear fractional relaxation differential equation

$$
\left\{\begin{array}{l}
{ }^{C} D^{\alpha} x(t)+w x(t)=f(t, x(t)), \quad 0<t \leq 1, \\
x(0)=x_{0}>0,
\end{array}\right.
$$

where $0<\alpha \leq 1, w>0$ and $f:[0,1] \times[0, \infty) \rightarrow[0, \infty)$ is a given continuous function. By using the method of the upper and lower solutions and Schauder and Banach fixed point theorems, the existence and uniqueness of solutions has been established.

Ahmad and Ntouyas in [3] studied the existence and uniqueness of solutions to the following boundary value problem

$$
\left\{\begin{array}{l}
\mathfrak{D}_{1}^{\alpha}\left(\mathfrak{D}_{1}^{\beta} u(t)-g\left(t, u_{t}\right)\right)=f\left(t, u_{t}\right), \quad t \in[1, b] \\
u(t)=\phi(t), \quad t \in[1-r, 1], \\
\mathfrak{D}_{1}^{\beta} u(1)=\eta \in \mathbb{R}
\end{array}\right.
$$

where $\mathfrak{D}_{1}^{\alpha}$ and $\mathfrak{D}_{1}^{\beta}$ are the Caputo-Hadamard fractional derivatives, $0<\alpha, \beta<1$. By employing the fixed point theorems, the authors obtained existence and uniqueness results.

In this paper, we are interested in the analysis of qualitative theory of the problems of the positive solutions to fractional differential equations. Inspired and motivated by the works mentioned above and the papers [1]-[13], [16] and the references therein, 
we concentrate on the positivity of solutions for the first-order nonlinear CaputoHadamard fractional relaxation differential equation

$$
\left\{\begin{array}{l}
\mathfrak{D}_{1}^{\alpha}(x(t)-g(t, x(t)))+w x(t)=f(t, x(t)), \quad 1<t \leq e, \\
x(1)=x_{0}>g\left(1, x_{0}\right)>0,
\end{array}\right.
$$

where $0<\alpha \leq 1, w>0, g, f:[1, e] \times[0, \infty) \rightarrow[0, \infty)$ are continuous. To show the existence and uniqueness of the positive solution, we transform (1.1) into an integral equation and then by the method of upper and lower solutions and use the Krasnoselskii and Banach fixed point theorems.

This paper is organized as follows. In Section 2, we introduce some notations and lemmas, and state some preliminaries results needed in later sections. Also, we present the inversion of (1.1) and the Banach and Krasnoselskii fixed point theorems. For details on the Banach and Krasnoselskii theorems we refer the reader to [15]. In Sections 3 and 4, we give and prove our main results on positivity and we provide an example to illustrate our results.

\section{Preliminaries}

Let $X=C([1, e])$ be the Banach space of all real-valued continuous functions defined on the compact interval $[1, e]$, endowed with the maximum norm. Define the cone

$$
\mathcal{E}=\{x \in X: x(t) \geq 0 \text { for all } t \in[1, e]\} .
$$

We introduce some necessary definitions, lemmas and theorems which will be used in this paper. For more details, see $[9,13]$.

Definition 2.1 ([9]). The Hadamard fractional integral of order $\alpha>0$ for a continuous function $x:[1,+\infty) \rightarrow \mathbb{R}$ is defined as

$$
\mathfrak{I}_{1}^{\alpha} x(t)=\frac{1}{\Gamma(\alpha)} \int_{1}^{t}\left(\log \frac{t}{s}\right)^{\alpha-1} x(s) \frac{d s}{s}, \quad \alpha>0 .
$$

Definition $2.2([9])$. The Caputo-Hadamard fractional derivative of order $\alpha>0$ for a continuous function $x:[1,+\infty) \rightarrow \mathbb{R}$ is defined as

$$
\mathfrak{D}_{1}^{\alpha} x(t)=\frac{1}{\Gamma(n-\alpha)} \int_{1}^{t}\left(\log \frac{t}{s}\right)^{n-\alpha-1} \delta^{n}(x)(s) \frac{d s}{s}, \quad n-1<\alpha<n,
$$

where $\delta^{n}=\left(t \frac{d}{d t}\right)^{n}, n \in \mathbb{N}$.

Lemma $2.1([9])$. Let $n-1<\alpha \leq n, n \in \mathbb{N}$ and $x \in C^{n}([1, T])$. Then

$$
\left(\mathfrak{I}_{1}^{\alpha} \mathfrak{D}_{1}^{\alpha} x\right)(t)=x(t)-\sum_{k=0}^{n-1} \frac{x^{(k)}(1)}{\Gamma(k+1)}(\log t)^{k} .
$$

Lemma $2.2([9])$. For all $\mu>0$ and $\nu>-1$

$$
\frac{1}{\Gamma(\mu)} \int_{1}^{t}\left(\log \frac{t}{s}\right)^{\mu-1}(\log s)^{\nu} \frac{d s}{s}=\frac{\Gamma(\nu+1)}{\Gamma(\mu+\nu+1)}(\log t)^{\mu+\nu} .
$$


Definition 2.3 ([14]). The two-parameter function of the Mittag-Leffler type is defined by the series expansion

$$
E_{\alpha, \beta}(z)=\sum_{n=0}^{\infty} \frac{z^{n}}{\Gamma(\alpha n+\beta)}, \quad \alpha>0, \beta \in \mathbb{C}, z \in \mathbb{C} .
$$

For $\beta=1$, we obtain the Mittag-Leffler function in one parameter

$$
E_{\alpha}(z)=\sum_{n=0}^{\infty} \frac{z^{n}}{\Gamma(\alpha n+1)}, \quad \alpha>0, z \in \mathbb{C} .
$$

Lemma 2.3 ([14]). The generalized Mittag-Leffler function $E_{\alpha, \beta}(-x)$ with $x \geq 0$ is completely monotonic if and only if $0<\alpha \leq 1$ and $\beta \geq \alpha$. In other words, it yields

$$
(-1)^{n} \frac{d^{n}}{d x^{n}} E_{\alpha, \beta}(-x) \geq 0, \quad \text { for all } n \in \mathbb{N} .
$$

Obviously, $0 \leq E_{\alpha, \beta}(-x) \leq \frac{1}{\Gamma(\beta)}$, where $x \geq 0,0 \leq \alpha \leq 1$ and $\beta \geq \alpha$.

The following lemma is fundamental to our results.

Lemma 2.4. Let $x \in C([1, e]), x^{\prime}$ and $\frac{\partial g}{\partial t}$ exist, then $x$ is a solution of $(1.1)$ if and only if

$$
\begin{aligned}
x(t)= & \left(x_{0}-g\left(1, x_{0}\right)\right) E_{\alpha}\left(-w(\log t)^{\alpha}\right)+g(t, x(t)) \\
& +\int_{1}^{t}\left(\log \frac{t}{s}\right)^{\alpha-1} E_{\alpha, \alpha}\left(-w\left(\log \frac{t}{s}\right)^{\alpha}\right) F(s, x(s)) \frac{d s}{s}, \quad 1 \leq t \leq e,
\end{aligned}
$$

where $F(t, x)=f(t, x)-w g(t, x)$.

Proof. It is easy to prove by the Laplace transform.

Lastly in this section, we state the fixed point theorems which enable us to prove the existence and uniqueness of a positive solution of (1.1).

Definition 2.4. Let $(X,\|\cdot\|)$ be a Banach space and $\mathcal{H}: X \rightarrow X$. The operator $\mathcal{H}$ is a contraction operator if there is an $\lambda \in(0,1)$ such that $x, y \in X$ imply

$$
\|\mathcal{H} x-\mathcal{H} y\| \leq \lambda\|x-y\| .
$$

Theorem 2.1 (Banach [15]). Let $\mathcal{K}$ be a nonempty closed convex subset of a Banach space $X$ and $\mathcal{H}: \mathcal{K} \rightarrow \mathcal{K}$ be a contraction operator. Then there is a unique $x \in \mathcal{K}$ with $\mathcal{H} x=x$.

Theorem 2.2 (Krasnoselskii fixed point theorem [15]). If $\mathcal{K}$ is a nonempty bounded, closed and convex subset of a Banach space $X, \mathcal{A}$ and $\mathcal{B}$ two operators defined on $\mathcal{K}$ with values in $X$ such that
i) $\mathcal{A} x+\mathcal{B} y \in \mathcal{K}$ for all $x, y \in \mathcal{K}$;
ii) $\mathcal{A}$ is continuous and compact;
iii) $\mathcal{B}$ is a contraction.

Then there exists $z \in \mathcal{K}$ such that $z=\mathcal{A} z+\mathcal{B} z$. 


\section{Existence of Positive Solutions}

In this section, we consider the results of existence problem for many cases of (1.1). Moreover, we introduce the following conditions.

$(H 1) g, F:[1, e] \times[0, \infty) \rightarrow[0, \infty)$ are continuous functions and $g$ is nondecreasing on $x$.

(H2) There exists $L_{g} \in(0,1)$ such that

$$
|g(t, x)-g(t, y)| \leq L_{g}\|x-y\| .
$$

(H3) There exists $L_{F}>0$ such that

$$
|F(t, x)-F(t, y)| \leq L_{F}\|x-y\| .
$$

We note that to apply Theorem 2.2 we need to construct two mappings, one is contraction and the other is completely continuous. Therefore, we express (2.1) as

$$
x(t)=(\mathcal{A} x)(t)+(\mathcal{B} x)(t)=(\mathcal{H} x)(t),
$$

where the operators $\mathcal{A}, \mathcal{B}: \mathcal{E} \rightarrow X$ are defined by

$$
(\mathcal{A} x)(t)=\int_{1}^{t}\left(\log \frac{t}{s}\right)^{\alpha-1} E_{\alpha, \alpha}\left(-w\left(\log \frac{t}{s}\right)^{\alpha}\right) F(s, x(s)) \frac{d s}{s}
$$

and

$$
(\mathcal{B} x)(t)=\left(x_{0}-g\left(1, x_{0}\right)\right) E_{\alpha}\left(-w(\log t)^{\alpha}\right)+g(t, x(t)) .
$$

We need the following lemmas to establish our results.

Lemma 3.1. Assume that (H1) holds. Then, the operator $\mathcal{A}: \mathcal{E} \rightarrow \mathcal{E}$ is completely continuous.

Proof. By Lemma 2.3 and taking into account that $F$ is continuous nonnegative function, we get that $\mathcal{A}: \mathcal{E} \rightarrow \mathcal{E}$ is continuous. The function $F:[1, e] \times B_{\eta} \rightarrow[0, \infty)$ is bounded, then there exists $\rho>0$ such that $0 \leq F(t, x(t)) \leq \rho$, where $B_{\eta}=$ $\{x \in \mathcal{E},\|x\| \leq \eta\}$. We obtain

$$
\begin{aligned}
|(\mathcal{A} x)(t)| & =\left|\int_{1}^{t}\left(\log \frac{t}{s}\right)^{\alpha-1} E_{\alpha, \alpha}\left(-w\left(\log \frac{t}{s}\right)^{\alpha}\right) F(s, x(s)) \frac{d s}{s}\right| \\
& \leq \frac{1}{\Gamma(\alpha)} \int_{1}^{t}\left(\log \frac{t}{s}\right)^{\alpha-1}|F(s, x(s))| \frac{d s}{s} \\
& \leq \frac{\rho}{\Gamma(\alpha)} \int_{1}^{t}\left(\log \frac{t}{s}\right)^{\alpha-1} \frac{d s}{s} \\
& \leq \frac{\rho(\log t)^{\alpha}}{\Gamma(\alpha+1)}
\end{aligned}
$$

Thus,

$$
\|\mathcal{A} x\| \leq \frac{\rho}{\Gamma(\alpha+1)}
$$

Hence, $\mathcal{A}\left(B_{\eta}\right)$ is uniformly bounded. 
Now, we will prove that $\mathcal{A}\left(B_{\eta}\right)$ is equicontinuous. Let $x \in B_{\eta}$, then for any $t_{1}, t_{2} \in[1, e], t_{2}>t_{1}$, we have

$$
\begin{aligned}
& \left|(\mathcal{A} x)\left(t_{1}\right)-(\mathcal{A} x)\left(t_{2}\right)\right| \\
= & \mid \int_{1}^{t_{1}}\left(\log \frac{t_{1}}{s}\right)^{\alpha-1} E_{\alpha, \alpha}\left(-w\left(\log \frac{t_{1}}{s}\right)^{\alpha}\right) F(s, x(s)) \frac{d s}{s} \\
& -\int_{1}^{t_{2}}\left(\log \frac{t_{2}}{s}\right)^{\alpha-1} E_{\alpha, \alpha}\left(-w\left(\log \frac{t_{2}}{s}\right)^{\alpha}\right) F(s, x(s)) \frac{d s}{s} \mid \\
\leq & \frac{1}{\Gamma(\alpha)} \int_{1}^{t_{1}}\left|\left(\log \frac{t_{1}}{s}\right)^{\alpha-1}-\left(\log \frac{t_{2}}{s}\right)^{\alpha-1}\right||F(s, x(s))| \frac{d s}{s} \\
& +\frac{1}{\Gamma(\alpha)} \int_{t_{1}}^{t_{2}}\left(\log \frac{t_{2}}{s}\right)^{\alpha-1}|F(s, x(s))| \frac{d s}{s} \\
\leq & \frac{\rho}{\Gamma(\alpha)}\left(\int_{1}^{t_{1}}\left(\left(\log \frac{t_{1}}{s}\right)^{\alpha-1}-\left(\log \frac{t_{2}}{s}\right)^{\alpha-1}\right) \frac{d s}{s}+\int_{t_{1}}^{t_{2}}\left(\log \frac{t_{2}}{s}\right)^{\alpha-1} \frac{d s}{s}\right) \\
\leq & \frac{\rho}{\Gamma(\alpha+1)}\left(\left(\log t_{1}\right)^{\alpha}-\left(\log t_{2}\right)^{\alpha}+2\left(\log \frac{t_{2}}{t_{1}}\right)^{\alpha}\right) \\
\leq & \frac{2 \rho}{\Gamma(\alpha+1)}\left(\log \frac{t_{2}}{t_{1}}\right)^{\alpha},
\end{aligned}
$$

which is independent of $x$ and tends to zero as $t_{2} \rightarrow t_{1}$. Thus, $\mathcal{A}\left(B_{\eta}\right)$ is equicontinuous. So, the compactness of $\mathcal{A}$ follows by Ascoli Arzela's theorem.

Lemma 3.2. Assume that $(H 1)$ and $(H 2)$ hold. Then the operator $\mathcal{B}: \mathcal{E} \rightarrow \mathcal{E}$ is a contraction.

Proof. By Lemma 2.3 and taking into account that $g$ is continuous nonnegative function and $x_{0}>g\left(1, x_{0}\right)$, we get that $\mathcal{B}: \mathcal{E} \rightarrow \mathcal{E}$. For $x, y \in \mathcal{E}$ we have

$$
|(\mathcal{B} x)(t)-(\mathcal{B} y)(t)|=|g(t, x(t))-g(t, y(t))| \leq L_{g}\|x-y\| .
$$

Thus, $\|\mathcal{B} x-\mathcal{B} y\| \leq L_{g}\|x-y\|$. Hence, $\mathcal{B}$ is a contraction.

Now, for any $x \in[a, b] \subset \mathbb{R}^{+}$, we define respectively the upper and lower control functions as follows

$$
H(t, x)=\sup _{a \leq y \leq x} F(t, y), \quad h(t, x)=\inf _{x \leq y \leq b} F(t, y) .
$$

It is clear that these functions are nondecreasing on $[a, b]$.

Definition 3.1. Let $\bar{x}, \underline{x} \in \mathcal{E}, a \leq \underline{x} \leq \bar{x} \leq b$, satisfying

$$
\begin{aligned}
\bar{x}(t) \geq & \left(x_{0}-g\left(1, x_{0}\right)\right) E_{\alpha}\left(-w(\log t)^{\alpha}\right)+g(t, \bar{x}(t)) \\
& +\int_{1}^{t}\left(\log \frac{t}{s}\right)^{\alpha-1} E_{\alpha, \alpha}\left(-w\left(\log \frac{t}{s}\right)^{\alpha}\right) H(s, \bar{x}(s)) \frac{d s}{s}, \quad 1 \leq t \leq e,
\end{aligned}
$$


and

$$
\begin{aligned}
\underline{x}(t) \leq & \left(x_{0}-g\left(1, x_{0}\right)\right) E_{\alpha}\left(-w(\log t)^{\alpha}\right)+g(t, \underline{x}(t)) \\
& +\int_{1}^{t}\left(\log \frac{t}{s}\right)^{\alpha-1} E_{\alpha, \alpha}\left(-w\left(\log \frac{t}{s}\right)^{\alpha}\right) h(s, \underline{x}(s)) \frac{d s}{s}, \quad 1 \leq t \leq e .
\end{aligned}
$$

Then the functions $\bar{x}$ and $\underline{x}$ are called a pair of upper and lower solutions for the equation (1.1).

Theorem 3.1. Assume that $(H 1)$ and $(H 2)$ hold and $\bar{x}$ and $\underline{x}$ are respectively upper and lower solutions of (1.1), then (1.1) has at least one positive solution.

Proof. Let

$$
\mathcal{K}=\{x \in \mathcal{E}: \underline{x}(t) \leq x(t) \leq \bar{x}(t), t \in[1, e]\} .
$$

As $\mathcal{K} \subset E$ and $\mathcal{K}$ is a nonempty bounded, closed and convex subset. By Lemma $3.1, \mathcal{A}: \mathcal{K} \rightarrow \mathcal{E}$ is completely continuous. Also, from Lemma 3.2, $\mathcal{B}: \mathcal{K} \rightarrow \mathcal{E}$ is a contraction. Next, we show that if $x, y \in \mathcal{K}$, we have $\mathcal{A} x+\mathcal{B} y \in \mathcal{K}$. For any $x, y \in \mathcal{K}$, we have $\underline{x} \leq x, y \leq \bar{x}$, then

$$
\begin{aligned}
& (\mathcal{A} x)(t)+(\mathcal{B} y)(t) \\
= & \left(x_{0}-g\left(1, x_{0}\right)\right) E_{\alpha}\left(-w(\log t)^{\alpha}\right)+g(t, y(t)) \\
& +\int_{1}^{t}\left(\log \frac{t}{s}\right)^{\alpha-1} E_{\alpha, \alpha}\left(-w\left(\log \frac{t}{s}\right)^{\alpha}\right) F(s, x(s)) \frac{d s}{s} \\
\leq & \left(x_{0}-g\left(1, x_{0}\right)\right) E_{\alpha}\left(-w(\log t)^{\alpha}\right)+g(t, \bar{x}(t)) \\
& +\int_{1}^{t}\left(\log \frac{t}{s}\right)^{\alpha-1} E_{\alpha, \alpha}\left(-w\left(\log \frac{t}{s}\right)^{\alpha}\right) H(s, \bar{x}(s)) \frac{d s}{s} \\
\leq & \bar{x}(t)
\end{aligned}
$$

and

$$
\begin{aligned}
& (\mathcal{A} x)(t)+(\mathcal{B} y)(t) \\
= & \left(x_{0}-g\left(1, x_{0}\right)\right) E_{\alpha}\left(-w(\log t)^{\alpha}\right)+g(t, y(t)) \\
& +\int_{1}^{t}\left(\log \frac{t}{s}\right)^{\alpha-1} E_{\alpha, \alpha}\left(-w\left(\log \frac{t}{s}\right)^{\alpha}\right) F(s, x(s)) \frac{d s}{s} \\
\geq & \left(x_{0}-g\left(1, x_{0}\right)\right) E_{\alpha}\left(-w(\log t)^{\alpha}\right)+g(t, \underline{x}(t)) \\
& +\int_{1}^{t}\left(\log \frac{t}{s}\right)^{\alpha-1} E_{\alpha, \alpha}\left(-w\left(\log \frac{t}{s}\right)^{\alpha}\right) h(s, \underline{x}(s)) \frac{d s}{s} \\
\geq & \underline{x}(t) .
\end{aligned}
$$

Thus, from (3.2) and (3.3), we obtain that $\mathcal{A} x+\mathcal{B} y \in \mathcal{K}$. We now see that all the conditions of the Krasnoselskii's fixed point theorem are satisfied. Thus there exists a fixed point $x$ in $\mathcal{K}$. Therefore, (1.1) has at least one positive solution $x$ in $\mathcal{K}$. 
Corollary 3.1. Assume that (H1) and (H2) hold and there exist $\lambda_{1}, \lambda_{2}, \lambda_{3}, \lambda_{4} \geq 0$ such that

$$
\lambda_{1} \leq g(t, x) \leq \lambda_{2}, \quad(t, x) \in[1, e] \times[0,+\infty)
$$

and

$$
\lambda_{3} \leq F(t, x) \leq \lambda_{4}, \quad(t, x) \in[1, e] \times[0,+\infty) .
$$

Then (1.1) has at least one positive solution $x \in \mathcal{E}$, moreover

$$
x(t) \geq\left(x_{0}-g\left(1, x_{0}\right)\right) E_{\alpha}\left(-w(\log t)^{\alpha}\right)+\lambda_{1}+\lambda_{3}(\log t)^{\alpha} E_{\alpha, \alpha+1}\left(-w(\log t)^{\alpha}\right)
$$

and

(3.7) $x(t) \leq\left(x_{0}-g\left(1, x_{0}\right)\right) E_{\alpha}\left(-w(\log t)^{\alpha}\right)+\lambda_{2}+\lambda_{4}(\log t)^{\alpha} E_{\alpha, \alpha+1}\left(-w(\log t)^{\alpha}\right)$.

Proof. From (3.5) and the definition of control functions, we have

$$
\lambda_{3} \leq h(t, x) \leq H(t, x) \leq \lambda_{4} .
$$

Now, let

$$
\begin{aligned}
\bar{x}(t)= & \left(x_{0}-g\left(1, x_{0}\right)\right) E_{\alpha}\left(-w(\log t)^{\alpha}\right)+\lambda_{2}+\lambda_{4}(\log t)^{\alpha} E_{\alpha, \alpha+1}\left(-w(\log t)^{\alpha}\right) \\
= & \left(x_{0}-g\left(1, x_{0}\right)\right) E_{\alpha}\left(-w(\log t)^{\alpha}\right)+\lambda_{2} \\
& +\lambda_{4} \int_{1}^{t}\left(\log \frac{t}{s}\right)^{\alpha-1} E_{\alpha, \alpha}\left(-w\left(\log \frac{t}{s}\right)^{\alpha}\right) \frac{d s}{s} .
\end{aligned}
$$

Taking into account (3.4) and (3.8), we have

$$
\begin{aligned}
\bar{x}(t)= & \left(x_{0}-g\left(1, x_{0}\right)\right) E_{\alpha}\left(-w(\log t)^{\alpha}\right)+\lambda_{2} \\
& +\lambda_{4} \int_{1}^{t}\left(\log \frac{t}{s}\right)^{\alpha-1} E_{\alpha, \alpha}\left(-w\left(\log \frac{t}{s}\right)^{\alpha}\right) \frac{d s}{s} \\
\geq & \left(x_{0}-g\left(1, x_{0}\right)\right) E_{\alpha}\left(-w(\log t)^{\alpha}\right)+g(t, \bar{x}(t)) \\
& +\int_{1}^{t}\left(\log \frac{t}{s}\right)^{\alpha-1} E_{\alpha, \alpha}\left(-w\left(\log \frac{t}{s}\right)^{\alpha}\right) H(s, \bar{x}(s)) \frac{d s}{s} .
\end{aligned}
$$

It is clear that $\bar{x}$ is the upper solution of (1.1).

Now, let

$$
\begin{aligned}
\underline{x}(t)= & \left(x_{0}-g\left(1, x_{0}\right)\right) E_{\alpha}\left(-w(\log t)^{\alpha}\right)+\lambda_{1}+\lambda_{3}(\log t)^{\alpha} E_{\alpha, \alpha+1}\left(-w(\log t)^{\alpha}\right) \\
= & \left(x_{0}-g\left(1, x_{0}\right)\right) E_{\alpha}\left(-w(\log t)^{\alpha}\right)+\lambda_{1} \\
& +\lambda_{3} \int_{1}^{t}\left(\log \frac{t}{s}\right)^{\alpha-1} E_{\alpha, \alpha}\left(-w\left(\log \frac{t}{s}\right)^{\alpha}\right) \frac{d s}{s} .
\end{aligned}
$$

By (3.4), (3.8) and the same way that we used to search the upper solution, we conclude also that $\underline{x}$ is the lower solution of (1.1). Therefore, from Theorem 3.1, we conclude that (1.1) has at least one positive solution $x \in \mathcal{E}$ which verifies the inequalities (3.6) and (3.7). 
Corollary 3.2. Assume that (H1) and (H2) hold and there exists $a_{1}, a_{2}>0$ such that

$$
a_{1} \leq g(t, x), a_{2} \leq F(t, x), \quad(t, x) \in[1, e] \times[0,+\infty)
$$

and

$$
\lim _{x \rightarrow+\infty} g(t, x)<+\infty, \quad \lim _{x \rightarrow+\infty} F(t, x)<+\infty,
$$

then (1.1) has at least one positive solution.

Proof. By (3.10), there exist positive constants $N_{1}, N_{2}, R_{1}$ and $R_{2}$ such that

$$
g(t, x) \leq N_{1}, \quad \text { for any } x \geq R_{1}, t \in[1, e],
$$

and

$$
F(t, x) \leq N_{2}, \quad \text { for any } x \geq R_{2}, t \in[1, e] .
$$

Let $C_{1}=\max _{1 \leq t \leq e, 0 \leq x \leq R_{1}} g(t, x)$ and $C_{2}=\max _{1 \leq t \leq e, 0 \leq x \leq R_{2}} F(t, x)$. Then, by (3.11) and (3.12), we have

$$
a_{1} \leq g(t, x) \leq N_{1}+C_{1}, \quad \text { for any } x \geq 0, t \in[1, e]
$$

and

$$
a_{2} \leq F(t, x) \leq N_{2}+C_{2}, \quad \text { for any } x \geq 0, t \in[1, e] .
$$

Thus, from Corollary 3.1, (1.1) has at least one positive solution $x$ in $\mathcal{E}$ which satisfies the following inequalities

$$
x(t) \geq\left(x_{0}-g\left(1, x_{0}\right)\right) E_{\alpha}\left(-w(\log t)^{\alpha}\right)+a_{1}+a_{2}(\log t)^{\alpha} E_{\alpha, \alpha+1}\left(-w(\log t)^{\alpha}\right)
$$

and

$$
\begin{aligned}
x(t) \leq & \left(x_{0}-g\left(1, x_{0}\right)\right) E_{\alpha}\left(-w(\log t)^{\alpha}\right)+N_{1}+C_{1} \\
& +\left(N_{2}+C_{2}\right)(\log t)^{\alpha} E_{\alpha, \alpha+1}\left(-w(\log t)^{\alpha}\right) .
\end{aligned}
$$

\section{Uniqueness of Positive Solution}

In this section, we shall prove the uniqueness of the positive solution using the contraction mapping principle.

Theorem 4.1. Assume that (H1)-(H3) hold and

$$
L_{g}+\frac{L_{F}}{\Gamma(\alpha+1)}<1
$$

then (1.1) has a unique positive solution $x \in \mathcal{K}$. 
Proof. From Theorem 3.1, it follows that (1.1) has at least one positive solution in $\mathcal{K}$. Hence, we need only to prove that the operator $\mathcal{H}$ defined in (3.1) is a contraction on $X$. In fact, since for any $x_{1}, x_{2} \in \mathcal{K},(H 2)$ and (H3) are verified, then we have

$$
\begin{aligned}
& \left|\left(\mathcal{H} x_{1}\right)(t)-\left(\mathcal{H} x_{2}\right)(t)\right| \\
\leq & \left|g\left(t, x_{1}(t)\right)-g\left(t, x_{2}(t)\right)\right| \\
& +\int_{1}^{t}\left(\log \frac{t}{s}\right)^{\alpha-1} E_{\alpha, \alpha}\left(-w\left(\log \frac{t}{s}\right)^{\alpha}\right)\left|F\left(s, x_{1}(s)\right)-F\left(s, x_{2}(s)\right)\right| \frac{d s}{s} \\
\leq & L_{g}\left\|x_{1}-x_{2}\right\|+\frac{(\log t)^{\alpha}}{\Gamma(\alpha+1)} L_{F}\left\|x_{1}-x_{2}\right\| \\
\leq & \left(L_{g}+\frac{L_{F}}{\Gamma(\alpha+1)}\right)\left\|x_{1}-x_{2}\right\| .
\end{aligned}
$$

Thus,

$$
\left\|\mathcal{H} x_{1}-\mathcal{H} x_{2}\right\| \leq\left(L_{g}+\frac{L_{F}}{\Gamma(\alpha+1)}\right)\left\|x_{1}-x_{2}\right\| .
$$

Hence, the operator $\mathcal{H}$ is a contraction mapping by (4.1). Therefore, by the contraction mapping principle, we conclude that the problem (1.1) has a unique positive solution $x \in \mathcal{K}$.

Finally, we give an example to illustrate our results.

Example 4.1. We consider the following nonlinear Caputo-Hadamard fractional relaxation differential equation

$$
\left\{\begin{array}{l}
\mathfrak{D}_{1}^{1 / 3}\left(x(t)-\frac{x(t)+2}{x(t)+3}\right)+x(t) \\
=\frac{(t+6) x^{2}(t)+(4 t+21) x(t)+5 t+18}{(t+3)\left(x^{2}(t)+4 x(t)+3\right)}, \quad 1<t \leq e \\
x(1)=1,
\end{array}\right.
$$

where

$$
\begin{aligned}
\alpha & =\frac{1}{3}, \quad w=1, \quad x_{0}=1, \quad g(t, x)=\frac{x+2}{x+3}, \quad g\left(1, x_{0}\right)=\frac{3}{4}, \\
f(t, x) & =\frac{(t+6) x^{2}+(4 t+21) x+5 t+18}{(t+3)\left(x^{2}+4 x+3\right)}, \quad F(t, x)=\frac{1}{3+t}\left(\frac{t}{x+1}+3\right) .
\end{aligned}
$$

Since $g$ is nondecreasing on $x$ and $F$ is decreasing on $x$

$$
\frac{2}{3} \leq g(t, x) \leq 1, \quad \frac{3}{3+e} \leq F(t, x) \leq 1
$$

for $(t, x) \in[1, e] \times[0, \infty)$. Hence, by Corollary 3.1, (4.2) has a positive solution, which verifies $\underline{x}(t) \leq x(t) \leq \bar{x}(t)$, where

$$
\bar{x}(t)=\frac{1}{4} E_{1 / 3}\left(-(\log t)^{1 / 3}\right)+1+(\log t)^{1 / 3} E_{1 / 3,4 / 3}\left(-(\log t)^{1 / 3}\right)
$$


and

$$
\underline{x}(t)=\frac{1}{4} E_{1 / 3}\left(-(\log t)^{1 / 3}\right)+\frac{2}{3}+\frac{3}{3+e}(\log t)^{1 / 3} E_{1 / 3,4 / 3}\left(-(\log t)^{1 / 3}\right),
$$

are respectively the upper and lower solutions of (4.2). Also, we have

$$
L_{g}+\frac{L_{F}}{\Gamma(\alpha+1)} \simeq 0.64<1
$$

Then, by Theorem 4.1, (4.2) has a unique positive solution which is bounded by $\underline{x}$ and $\bar{x}$.

\section{REFERENCES}

[1] S. Abbas, Existence of solutions to fractional order ordinary and delay differential equations and applications, Electron. J. Differential Equations 2011(9) (2011), 1-11.

[2] R. P. Agarwal, Y. Zhou and Y. He, Existence of fractional functional differential equations, Comput. Math. Appl. 59 (2010), 1095-1100.

[3] B. Ahmad and S. K. Ntouyas, Existence and uniqueness of solutions for Caputo-Hadamard sequential fractional order neutral functional differential equations, Electron. J. Differential Equations $\mathbf{2 0 1 7}(36)$ (2017), 1-11.

[4] H. Boulares, A. Ardjouni and Y. Laskri, Positive solutions for nonlinear fractional differential equations, Positivity 21 (2017), 1201-1212.

[5] H. Boulares, A. Ardjouni and Y. Laskri, Stability in delay nonlinear fractional differential equations, Rend. Circ. Mat. Palermo 65 (2016), 243-253.

[6] A. Chidouh, A. Guezane-Lakoud and R. Bebbouchi, Positive solutions of the fractional relaxation equation using lower and upper solutions, Vietnam J. Math. 44(4) (2016), 739-748.

[7] F. Ge and C. Kou, Stability analysis by Krasnoselskii's fixed point theorem for nonlinear fractional differential equations, Appl. Math. Comput. 257 (2015), 308-316.

[8] F. Ge and C. Kou, Asymptotic stability of solutions of nonlinear fractional differential equations of order $1<\alpha<2$, Journal of Shanghai Normal University 44(3) (2015), 284-290.

[9] A. A. Kilbas, H. H. Srivastava and J. J. Trujillo, Theory and Applications of Fractional Differential Equations, Elsevier Science, Amsterdam, 2006.

[10] C. Kou, H. Zhou and Y. Yan, Existence of solutions of initial value problems for nonlinear fractional differential equations on the half-axis, Nonlinear Anal. 74 (2011), 5975-5986.

[11] V. Lakshmikantham and A. S. Vatsala, Basic theory of fractional differential equations, Nonlinear Anal. 69 (2008) 2677-2682.

[12] N. Li and C. Wang, New existence results of positive solution for a class of nonlinear fractional differential equations, Acta Math. Sci. 33 (2013), 847-854.

[13] I. Podlubny, Fractional Differential Equations, Academic Press, San Diego, 1999.

[14] W. R. Schneider, Completely monotone generalized Mittag-Leffler functions, Expo. Math. 14 (1996), 3-16.

[15] D. R. Smart, Fixed Point Theorems, Cambridge Tracts in Mathematics 66, Cambridge University Press, London, New York, 1974.

[16] S. Zhang, The existence of a positive solution for a nonlinear fractional differential equation, J. Math. Anal. Appl. 252 (2000), 804-812. 
${ }^{1}$ Department of Mathematics and Informatics, University of Souk Ahras,

P.O. Box 1553, Souk Ahras, Algeria

Email address: abd_ardjouni@yahoo.fr

${ }^{2}$ Department of Mathematics, University OF ANNABA,

P.O. Box 12, Annaba, Algeria

Email address: adjoudi@yahoo.com 